\title{
Metabolism - New Therapeutic Targets for Inflammatory Bowel Disease
}

Genetic studies conducted over the last 20 years have been hugely successful in identifying variation across the genome that is associated with disease risk. The inflammatory bowel diseases (IBD) Crohn's disease and ulcerative colitis have been exemplars of this success, with now $>250$ individual genomic risk coordinates identified. This success sometimes overshadows the more humbling fact that mechanistic understanding is very scarce for how these genetic risk factors conspire with environmental triggers to cause disease. For some risk loci, we completely lack any understanding of the very basic function of their gene products.

An open reading frame on chromosome 13 (C13orf31) was one of these intriguing, entirely blank, loci. A coding variant, which leads to a valine-for-isoleucine substitution at amino acid position 254 (I254V) increases risk for Crohn's disease as well as for leprosy, a chronic infection with Mycobacterium leprae. Very rare deleterious loss-of-function variants detected in consanguineous families, such as an argininefor-cysteine substitution at amino acid 284 (C284R), cause autosomal recessive (i.e. monogenic) forms of very-early onset Crohn's disease, or, indeed the same variant, Still's disease. Still's disease (or systemic juvenile idiopathic arthritis, sJIA), where this gene is the sole known cause of its monogenic form, is the paradigm of autoinflammation-cum-autoimmunity. It starts in toddlers as an IL-1-mediated periodic fever syndrome, which morphs over weeks into a destructive, debilitating arthritis, which is thought to be T cell driven. The C-terminal half of the C13orf31 gene product is indeed evolutionarily highly conserved, and the X-ray crystallographic structures of several of its bacterial orthologues had been solved in the mid-2000s. These structures suggested that the protein was an enzyme. However, not even a sub-domain of this protein exhibited any homology to domains or proteins of known function, hence its function remained entirely enigmatic.
We discovered that this protein, which we named FAMIN [1], is an unprecedented, single pocket multi-functional purine nucleoside-metabolising enzyme, with its activities conserved from bacteria to man. Specifically, FAMIN irreversibly deaminates adenosine to inosine, and reversibly phosphorolytically cleaves inosine, guanosine and methyl-thio-adenosine into hypoxanthine, guanine, and adenine and their respective ribose-1-phosphates. These activities had been thought to be the domain of namesake enzymes ADA, PNP and MTAP in any form of life. Additionally, FAMIN directly cleaved adenosine into adenine, an activity that had been considered outrightly absent from eukaryotic life [2]. Since purine nucleotide de novo synthesis directly yields inosine monophosphate (IMP), from which adenosine and guanosine monophosphates (AMP, GMP) are generated, hitherto it had been thought that ADA, PNP and MTAP are the sole source of the nucleobases adenine, guanine and hypoxanthine. We solved the structure of FAMIN's bacterial orthologue YlmD in complex with its substrate inosine [2]. This demonstrated that inosine indeed binds to the predicted single pocket of the enzyme, with the ribose coordinated by a Cys-His-His triad via a zinc. Intriguingly, the orthologous cysteine in FAMIN is mutated in the loss-of-function variant (C284R) that causes monogenic Crohn's and Still's disease. We further demonstrated that the I254V variant is not only hypomorphic, but also leads to a qualitative catalytic change. Whilst FAMIN-254I prefers adenosine phosphorolysis to adenine, FAMIN-254V predominantly deaminates adenosine to inosine with onwards phosphorolytic cleavage to hypoxanthine. Adenosine, adenine and ribose are primordial metabolites from which life has emerged from prebiotic chemistry. The discovery of such multifunctional purine enzyme was particularly surprising, since central purine metabolism had been considered settled for the last half century.
This posited the question how such multifunctional purine enzyme would affect metabolic and immune function. We discovered that FAMIN enables a purine nucleotide cycle (PNC) in macrophages, in which the enzyme is particularly highly expressed. A PNC had originally been described by Lowenstein and Tornheim in extracts of skeletal muscle [3]. It consists of the cyclical deamination of AMP to IMP, and re-amination back to AMP via the intermediate succinyl-AMP. The re-amination is fuelled by GTP hydrolysis and consumes aspartate, whose carbons are then released as fumarate, which can be reversibly hydrated to malate. As such, the FAMIN-enables PNC controlled electron $\left(e^{-}\right)$ and proton $\left(\mathrm{H}^{+}\right)$transfer from cytoplasm, primarily from glycolysis, to mitochondria. Indeed, reduced or absent FAMIN activity results in gradual cytoplasmic acidification and reductive stress due to an increased $\mathrm{NADH} / \mathrm{NAD}^{+}$ratio in the cytoplasm. This is linked to a decrease in cells' extracellular acidification rate (ECAR), a measure of glycolytic activity as protons are co-exported from a cell with lactate, and a decrease in oxygen consumption rate (OCR), reflective of mitochondrial oxidative phosphorylation. Despite these profound metabolic perturbations, reduced or absent FAMIN activity does not have a transcriptional footprint, further arguing for a very ancient mechanism.

Hence the in-depth investigation of a single disease risk gene has revealed a fundamental biochemical function that is conserved from bacteria to man, with ramifications that go far beyond the associated diseases.

\section{Conflict of Interest}

The author declare that he has no conflict of interest. 
Author

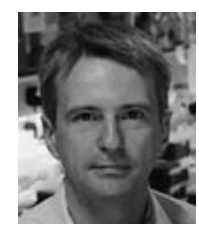

\section{Arthur Kaser}

FMedSci, Cambridge Institute of Therapeutic Immunology and Infectious Disease, and Division of Gastroenterology and Hepatology, Department of Medicine, University of Cambridge, Cambridge, United Kingdom

\section{Arthur Kaser FMedSci}

Cambridge Institute of Therapeutic Immunology and Infectious Disease, and Division of Gastroenterology and Hepatology

Department of Medicine

University of Cambridge

Puddicombe Way

Cambridge CB2 OAW

United Kingdom

ak729@cam.ac.uk

\section{References}

[1] Cader MZ, Boroviak K, Zhang Q et al. C13orf31 (FAMIN) is a central regulator of immunometabolic function. Nat Immunol 2016; 17: 1046-1056. doi:10.1038/ni.3532

[2] Cader MZ, Pereira de Almeida Rodrigues RP, West JA et al. FAMIN is a multifunctional purine enzyme enabling the purine nucleotide cycle. Cell 2020; 180: 278-295.e23. doi:10.1016/j.cell.2019.12.017

[3] Lowenstein J, Tornheim, K. Ammonia production in muscle: the purine nucleotide cycle. Science 1971; 171: 397-400. doi:10.1126/science.171.3969.397 